\title{
The Variational Calculus on Time Scales
}

\author{
Delfim F. M. Torres ${ }^{1, a}$ \\ ${ }^{1}$ Department of Mathematics, University of Aveiro, 3810-193 Aveiro, Portugal
}

\begin{abstract}
Received 10 December 2009, Accepted 15 February 2010
Abstract. The discrete, the quantum, and the continuous calculus of variations, have been recently unified and extended by using the theory of time scales. Such unification and extension is, however, not unique, and two approaches are followed in the literature: one dealing with minimization of delta integrals; the other dealing with minimization of nabla integrals. Here we review a more general approach to the calculus of variations on time scales that allows to obtain both delta and nabla results as particular cases.
\end{abstract}

Key words: time scales; delta and nabla derivatives and integrals; Euler-Lagrange equations; calculus of variations.

\section{Introduction}

The calculus on time scales is a recent field introduced by B. Aulbach and S. Hilger in order to unify the theories of difference and differential equations $[8,28]$. It has found applications in several contexts that require simultaneous modeling of discrete and continuous data, and is nowadays under strong current research in areas as diverse as control of population, quantum calculus, economics, communication networks and robotic control (see [1, 46] and references therein). The area of the calculus of variations on time scales, which we are concerned here, is in its beginning and is a fertile area of research [20]. As particular cases, one gets the classical calculus of variations [23], the discrete-time calculus of variations [30], and the $q$-calculus of variations [9].

The calculus of variations on time scales was introduced in 2004 by M. Bohner using the delta derivative and integral [12], and has been since then further developed by several different authors in several different directions $[2,11,21,22,33,34]$. In all these works, the integral functional to be extremized has the form

$$
\mathcal{J}_{\Delta}(y)=\int_{a}^{b} L\left(t, y^{\sigma}(t), y^{\Delta}(t)\right) \Delta t .
$$

Motivated by applications in economics [4, 7], a different formulation for the problems of the calculus of variations on time scales has been considered, which involve a functional with a nabla derivative and a nabla integral $[3,6,43]$ :

$$
\mathcal{J}_{\nabla}(y)=\int_{a}^{b} L\left(t, y^{\rho}(t), y^{\nabla}(t)\right) \nabla t .
$$

Formulations (1) and (2) are good in the sense that results obtained via delta and nabla approaches are similar among them and similar to the classical results of the calculus of variations. An example of this is given by the time scale versions of the Euler-Lagrange equations: if

\footnotetext{
${ }^{\mathrm{a} C}$ Corresponding author: delfim@ua.pt
}

$y \in C_{\text {rd }}^{2}$ is an extremizer of (1), then $y$ satisfies the deltadifferential equation

$$
\frac{\Delta}{\Delta t} \partial_{3} L\left(t, y^{\sigma}(t), y^{\Delta}(t)\right)=\partial_{2} L\left(t, y^{\sigma}(t), y^{\Delta}(t)\right)
$$

for all $t \in[a, b]^{\kappa^{2}}[12]$; if $y \in C_{\mathrm{ld}}^{2}$ is an extremizer of (2), then $y$ satisfies the nabla-differential equation

$$
\frac{\nabla}{\nabla t} \partial_{3} L\left(t, y^{\rho}(t), y^{\nabla}(t)\right)=\partial_{2} L\left(t, y^{\rho}(t), y^{\nabla}(t)\right)
$$

for all $t \in[a, b]_{\kappa^{2}}$ [43], where we use $\partial_{i} L$ to denote the standard partial derivative of $L(\cdot, \cdot, \cdot)$ with respect to its $i$ th variable, $i=1,2,3$. In the classical context $\mathbb{T}=\mathbb{R}$ one has

$$
\mathcal{J}_{\Delta}(y)=\mathcal{J}_{\nabla}(y)=\int_{a}^{b} L\left(t, y(t), y^{\prime}(t)\right) d t
$$

and both (3) and (4) coincide with the standard EulerLagrange equation: if $y \in C^{2}$ is an extremizer of the integral functional (5), then

$$
\frac{d}{d t} \partial_{3} L\left(t, y(t), y^{\prime}(t)\right)=\partial_{2} L\left(t, y(t), y^{\prime}(t)\right)
$$

for all $t \in[a, b]$. However, the problems of extremizing (1) and (2) are intrinsically different, in the sense that is not possible to obtain the nabla results as corollaries of the delta ones and vice versa. Indeed, if admissible functions $y$ are of class $C^{2}$ then (cf. [27])

$$
\begin{aligned}
\mathcal{J}_{\Delta}(y) & =\int_{a}^{b} L\left(t, y^{\sigma}(t), y^{\Delta}(t)\right) \Delta t \\
& =\int_{a}^{b} L\left(\rho(t),\left(y^{\sigma}\right)^{\rho}(t), y^{\nabla}(t)\right) \nabla t
\end{aligned}
$$

while

$$
\begin{aligned}
\mathcal{J}_{\nabla}(y) & =\int_{a}^{b} L\left(t, y^{\rho}(t), y^{\nabla}(t)\right) \nabla t \\
& =\int_{a}^{b} L\left(\sigma(t),\left(y^{\rho}\right)^{\sigma}(t), y^{\Delta}(t)\right) \Delta t
\end{aligned}
$$


and one easily see that functionals (1) and (2) have a different nature and are not compatible with each other.

In this paper we consider the more general delta-nabla formulation of the calculus of variations introduced in [37] and further developed in [24, 25, 26, 38], that includes, as trivial examples, the problems with functionals $\mathcal{J}_{\Delta}(y)$ and $\mathcal{J}_{\nabla}(y)$ that have been previously studied in the literature of time scales. For a different approach, based on the concept of duality [17], we refer the reader to $[42,44,45]$. One of the main results provide an Euler-Lagrange necessary optimality type condition for the more general integral functional $\mathcal{J}$ we are promoting here (Section 3.1). As straight corollaries we obtain both delta- and nabla-differential equations (3) and (4). Natural boundary conditions and necessary optimality conditions for delta-nabla isoperimetric problems of the calculus of variations are obtained as well (Sections 3.2 and 3.3, respectively). Simple illustrative examples on the application of the results are given in detail.

Let $\mathbb{T}$ be a given time scale with $a, b \in \mathbb{T}, a<b$, and $(\mathbb{T} \backslash\{a, b\}) \cap[a, b] \neq \emptyset ; L_{\Delta}(\cdot, \cdot, \cdot)$ and $L_{\nabla}(\cdot, \cdot, \cdot)$ be two given smooth functions from $\mathbb{T} \times \mathbb{R}^{2}$ to $\mathbb{R}$. The results here discussed are trivially generalized for admissible functions $y: \mathbb{T} \rightarrow \mathbb{R}^{n}$ but for simplicity of presentation we restrict ourselves to the scalar case $n=1$. We consider the delta-nabla integral functional

$$
\begin{aligned}
\mathcal{J}(y) & =\left(\int_{a}^{b} L_{\Delta}[y](t) \Delta t\right) \cdot\left(\int_{a}^{b} L_{\nabla}\{y\}(t) \nabla t\right) \\
& =\int_{a}^{b} \int_{a}^{b} L_{\Delta}[y](t) \cdot L_{\nabla}\{y\}(t) \Delta t \nabla \tau
\end{aligned}
$$

where, for simplicity of notation, we use the operators $[\cdot]$ and $\{\cdot\}$ defined by

$$
\begin{aligned}
{[y](t) } & =\left(t, y^{\sigma}(t), y^{\Delta}(t)\right), \\
\{y\}(t) & =\left(t, y^{\rho}(t), y^{\nabla}(t)\right) .
\end{aligned}
$$

Note that with the new operators we write (1) and (2) as

$$
\begin{aligned}
& \mathcal{J}_{\Delta}(y)=\int_{a}^{b} L_{\Delta}[y](t) \Delta t, \\
& \mathcal{J}_{\nabla}(y)=\int_{a}^{b} L_{\nabla}\{y\}(t) \nabla t .
\end{aligned}
$$

Remark 1. In the particular case $L_{\nabla} \equiv \frac{1}{b-a}$ functional (6) reduces to (1) (i.e., $\mathcal{J}(y)=\mathcal{J}_{\Delta}(y)$ ); in the particular case $L_{\Delta} \equiv \frac{1}{b-a}$ functional (6) reduces to (2) (i.e., $\mathcal{J}(y)=\mathcal{J}_{\nabla}(y)$ ).

Our main goal is to answer the following question: What is the Euler-Lagrange equation for $\mathcal{J}(y)$ defined by (6)?

\section{The time scales calculus}

The aim of the calculus on time scales is to unify continuous and discrete analysis into a general theory. The motivation for such general theory is rooted in the fact that many results concerning differential equations carry over quite easily to corresponding results for difference equations, while other results seem to be very different from continuous counterparts. The unification and extension given by the theory of time scales helps to explain such similarities and discrepancies.

In this section we introduce the basic definitions and results that will be needed in the sequel. For a more general presentation of the theory of time scales and detailed proofs, we refer the reader to the books [14, 15, 31].

As usual, $\mathbb{R}, \mathbb{Z}$, and $\mathbb{N}$ denote, respectively, the set of real, integer, and natural numbers.

\subsection{The delta calculus}

A time scale $\mathbb{T}$ is an arbitrary nonempty closed subset of $\mathbb{R}$. Besides standard cases of $\mathbb{R}$ (continuous time) and $\mathbb{Z}$ (discrete time), many different models of time may be used, e.g., the $h$-numbers $(\mathbb{T}=h \mathbb{Z}:=\{h z \mid z \in \mathbb{Z}\}$, where $h>0$ is a fixed real number) and the $q$-numbers $\left(\mathbb{T}=q^{\mathbb{N}_{0}}:=\left\{q^{k} \mid k \in \mathbb{N}_{0}\right\}\right.$, where $q>1$ is a fixed real number). We assume that a time scale $\mathbb{T}$ has the topology that it inherits from the real numbers with the standard topology. For each time scale $\mathbb{T}$ the following operators are used:

- the forward jump operator $\sigma: \mathbb{T} \rightarrow \mathbb{T}$, defined by $\sigma(t):=\inf \{s \in \mathbb{T}: s>t\}$ for $t \neq \sup \mathbb{T}$ and $\sigma(\sup \mathbb{T})=\sup \mathbb{T}$ if $\sup \mathbb{T}<+\infty$;

- the backward jump operator $\rho: \mathbb{T} \rightarrow \mathbb{T}$, defined by $\rho(t):=\sup \{s \in \mathbb{T}: s<t\}$ for $t \neq \inf \mathbb{T}$ and $\rho(\inf \mathbb{T})=\inf \mathbb{T}$ if inf $\mathbb{T}>-\infty$;

- the forward graininess function $\mu: \mathbb{T} \rightarrow[0, \infty)$, defined by $\mu(t):=\sigma(t)-t$;

- the backward graininess function $\nu: \mathbb{T} \rightarrow[0, \infty)$, defined by $\nu(t)=t-\rho(t)$.

Example 1. If $\mathbb{T}=\mathbb{R}$, then for any $t \in \mathbb{T} \sigma(t)=$ $\rho(t)=t$ and $\mu(t)=\nu(t)=0$. If $\mathbb{T}=h \mathbb{Z}, h>0$, then for every $t \in \mathbb{T} \sigma(t)=t+h, \rho(t)=t-h$, and $\mu(t)=\nu(t)=h$. On the other hand, if $\mathbb{T}=q^{\mathbb{N}_{0}}, q>1$, then we have $\sigma(t)=q t, \rho(t)=q^{-1} t, \mu(t)=(q-1) t$, and $\nu(t)=\left(1-q^{-1}\right) t$.

A point $t \in \mathbb{T}$ is called right-dense, right-scattered, left-dense or left-scattered if $\sigma(t)=t, \sigma(t)>t, \rho(t)=t$, and $\rho(t)<t$, respectively. We say that $t$ is isolated if $\rho(t)<t<\sigma(t)$, that $t$ is dense if $\rho(t)=t=\sigma(t)$.

If $\sup \mathbb{T}$ is finite and left-scattered, we define $\mathbb{T}^{\kappa}:=$ $\mathbb{T} \backslash\{\sup \mathbb{T}\}$, otherwise $\mathbb{T}^{\kappa}:=\mathbb{T}$. 
Definition 1. We say that a function $f: \mathbb{T} \rightarrow \mathbb{R}$ is delta differentiable at $t \in \mathbb{T}^{\kappa}$ if there exists a number $f^{\Delta}(t)$ such that for all $\varepsilon>0$ there is a neighborhood $U$ of $t$ such that

$$
\left|f(\sigma(t))-f(s)-f^{\Delta}(t)(\sigma(t)-s)\right| \leq \varepsilon|\sigma(t)-s|
$$

for all $s \in U$. We call $f^{\Delta}(t)$ the delta derivative of $f$ at $t$ and $f$ is said delta differentiable on $\mathbb{T}^{\kappa}$ provided $f^{\Delta}(t)$ exists for all $t \in \mathbb{T}^{\kappa}$.

Remark 2. If $t \in \mathbb{T} \backslash \mathbb{T}^{\kappa}$, then $f^{\Delta}(t)$ is not uniquely defined, since for such a point $t$, small neighborhoods $U$ of $t$ consist only of $t$ and, besides, we have $\sigma(t)=t$. For this reason, maximal left-scattered points are omitted in Definition 1.

Note that in right-dense points

$$
f^{\Delta}(t)=\lim _{s \rightarrow t} \frac{f(t)-f(s)}{t-s},
$$

provided this limit exists, and in right-scattered points

$$
f^{\Delta}(t)=\frac{f(\sigma(t))-f(t)}{\mu(t)},
$$

provided $f$ is continuous at $t$.

Example 2. If $\mathbb{T}=\mathbb{R}$, then $f: \mathbb{R} \rightarrow \mathbb{R}$ is delta differentiable at $t \in \mathbb{R}$ if and only if $f$ is differentiable in the ordinary sense at $t$. Then, $f^{\Delta}(t)=f^{\prime}(t)$. If $\mathbb{T}=h \mathbb{Z}$, $h>0$, then $f: \mathbb{Z} \rightarrow \mathbb{R}$ is always delta differentiable at every $t \in \mathbb{Z}$ with $f^{\Delta}(t)=\frac{f(t+h)-f(t)}{h}$. If $\mathbb{T}=q^{\mathbb{N}_{0}}$, $q>1$, then $f^{\Delta}(t)=\frac{f(q t)-f(t)}{(q-1) t}$, i.e., we get the usual $q$ derivative of quantum calculus [29]. For a more general quantum calculus see [16, 39].

Delta derivatives of higher order are defined in the standard way. Let $r \in \mathbb{N}, \mathbb{T}^{\kappa^{0}}:=\mathbb{T}$, and $\mathbb{T}^{\kappa^{i}}:=$ $\left(\mathbb{T}^{\kappa^{i-1}}\right)^{\kappa}, i=1, \ldots, r$. For convenience we also put $f^{\Delta^{0}}=f$ and $f^{\Delta^{1}}=f^{\Delta}$. We define the $r^{t h}$-delta derivative $f^{\Delta^{r}}$ of $f$ provided $f^{\Delta^{r-1}}$ is delta differentiable on $\mathbb{T}^{\kappa^{r}}$ with derivative $f^{\Delta^{r}}=\left(f^{\Delta^{r-1}}\right)^{\Delta}: \mathbb{T}^{\kappa^{r}} \rightarrow \mathbb{R}$.

We shall often denote $f^{\Delta}$ by $\frac{\Delta}{\Delta t} f$ if $f$ is a composition of other functions of $t$. For $f: \mathbb{T} \rightarrow X$, where $X$ is an arbitrary set, we define $f^{\sigma}:=f \circ \sigma$. hold:

For delta differentiable $f$ and $g$, the next formulas

$$
\begin{aligned}
f^{\sigma}(t) & =f(t)+\mu(t) f^{\Delta}(t), \\
(f g)^{\Delta}(t) & =f^{\Delta}(t) g^{\sigma}(t)+f(t) g^{\Delta}(t) \\
& =f^{\Delta}(t) g(t)+f^{\sigma}(t) g^{\Delta}(t) .
\end{aligned}
$$

Let $a, b \in \mathbb{T}, a<b$. We define the interval $[a, b]$ in $\mathbb{T}$ by

$$
[a, b]:=\{t \in \mathbb{T}: a \leq t \leq b\} .
$$

Open intervals, half-open intervals and unbounded intervals in $\mathbb{T}$ are defined accordingly.

In order to describe a class of functions that possess a delta antiderivative, the following definition is introduced:

Definition 2. A function $f: \mathbb{T} \rightarrow \mathbb{R}$ is called rdcontinuous if it is continuous at right-dense points in $\mathbb{T}$ and its left-sided limits exist (finite) at left-dense points in $\mathbb{T}$. We denote the set of all rd-continuous functions by $C_{r d}^{0}=C_{r d}=C_{r d}(\mathbb{T})=C_{r d}(\mathbb{T} ; \mathbb{R})$. The set of functions $f: \mathbb{T} \rightarrow \mathbb{R}$ that are delta differentiable and whose delta derivative is rd-continuous is denoted by $C_{r d}^{1}=C_{r d}^{1}(\mathbb{T})=C_{r d}^{1}(\mathbb{T} ; \mathbb{R})$. In general, we say that $f \in C_{r d}^{r}, r \in \mathbb{N}$, if $f^{\Delta} \in C_{r d}^{r-1}$.

Definition 3. A function $F: \mathbb{T} \rightarrow \mathbb{R}$ is called a delta antiderivative of $f: \mathbb{T} \rightarrow \mathbb{R}$ provided

$$
F^{\Delta}(t)=f(t), \quad \forall t \in \mathbb{T}^{\kappa} .
$$

In this case we define the delta integral of $f$ from $a$ to $b$ $(a, b \in \mathbb{T})$ by

$$
\int_{a}^{b} f(t) \Delta t:=F(b)-F(a) .
$$

Theorem 4 (Theorem 1.74 of [14]). Every rd-continuous function has a delta antiderivative. In particular, if a $\in$ $\mathbb{T}$, then the function $F$ defined by

$$
F(t)=\int_{a}^{t} f(\tau) \Delta \tau, \quad t \in \mathbb{T},
$$

is a delta antiderivative of $f$.

Example 3. Let $a, b \in \mathbb{T}$ with $a<b$ and $f \in C_{r d}$. (i) If $\mathbb{T}=\mathbb{R}$, then $\int_{a}^{b} f(t) \Delta t=\int_{a}^{b} f(t) d t$, where the integral on the right-hand side is the classical Riemann integral. (ii) If $\mathbb{T}=h \mathbb{Z}, h>0$, then

$$
\int_{a}^{b} f(t) \Delta t=\sum_{k=\frac{a}{h}}^{\frac{b}{h}-1} f(k h) h .
$$

If $\mathbb{T}=q^{\mathbb{N}_{0}}, q>1$, then

$$
\int_{a}^{b} f(t) \Delta t=(1-q) \sum_{t \in[a, b)} f(t) t
$$

(cf. the usual q-integral of quantum calculus [29]).

Theorem 5 (Theorems 1.75 and 1.77 of [14]). If $a, b, c \in$ $\mathbb{T}, a \leq c \leq b, \alpha \in \mathbb{R}$, and $f, g \in C_{r d}(\mathbb{T}, \mathbb{R})$, then

$$
\begin{aligned}
& \text { 1. } \int_{a}^{b}(f(t)+g(t)) \Delta t=\int_{a}^{b} f(t) \Delta t+\int_{a}^{b} g(t) \Delta t \\
& \text { 2. } \int_{a}^{b} \alpha f(t) \Delta t=\alpha \int_{a}^{b} f(t) \Delta t
\end{aligned}
$$


3. $\int_{a}^{b} f(t) \Delta t=-\int_{b}^{a} f(t) \Delta t$

4. $\int_{a}^{a} f(t) \Delta t=0$;

5. $\int_{a}^{b} f(t) \Delta t=\int_{a}^{c} f(t) \Delta t+\int_{c}^{b} f(t) \Delta t$

6. If $f(t)>0$ for all $a \leq t<b$, then $\int_{a}^{b} f(t) \Delta t>0$;

7. If $t \in \mathbb{T}^{\kappa}$, then $\int_{t}^{\sigma(t)} f(\tau) \Delta \tau=\mu(t) f(t)$.

We have presented only the very basic concepts of the theory of time scales. Nowadays the time scales theory covers nonlinear and higher order dynamic equations, boundary value problems, dynamic inequalities, symplectic dynamical systems, etc. An analogous theory has been later developed for the "nabla derivative", denoted $f^{\nabla}$, which is a generalization of the backward difference operator from discrete calculus $[5,14]$. This is the subject of our next section. The nabla calculus seems to be particularly useful as a modeling technique in the calculus of variations with applications to economics [4, 7, 32].

\subsection{The nabla approach}

In order to introduce the definition of nabla derivative, we define a new set $\mathbb{T}_{\kappa}$ which is derived from $\mathbb{T}$ as follows: if $\mathbb{T}$ has a right-scattered minimum $m$, then $\mathbb{T}_{\kappa}=\mathbb{T} \backslash\{m\}$; otherwise, $\mathbb{T}_{\kappa}=\mathbb{T}$. In order to simplify expressions, and similarly as done with composition with $\sigma$, we define $f^{\rho}(t):=f(\rho(t))$.

Definition 6. We say that a function $f: \mathbb{T} \rightarrow \mathbb{R}$ is nabla differentiable at $t \in \mathbb{T}_{\kappa}$ if there is a number $f^{\nabla}(t)$ such that for all $\varepsilon>0$ there exists a neighborhood $U$ of $t$ such that

$$
\left|f^{\rho}(t)-f(s)-f^{\nabla}(t)(\rho(t)-s)\right| \leq \varepsilon|\rho(t)-s|
$$

for all $s \in U$. We call $f^{\nabla}(t)$ the nabla derivative of $f$ at t. Moreover, we say that $f$ is nabla differentiable on $\mathbb{T}$ provided $f \nabla(t)$ exists for all $t \in \mathbb{T}_{\kappa}$.

Theorem 7. (Theorem 8.39 in [14]) Let $\mathbb{T}$ be a time scale, $f: \mathbb{T} \rightarrow \mathbb{R}$, and $t \in \mathbb{T}_{\kappa}$. The following holds:

1. If $f$ is nabla differentiable at $t$, then $f$ is continuous at $t$.

2. If $f$ is continuous at $t$ and $t$ is left-scattered, then $f$ is nabla differentiable at $t$ and

$$
f^{\nabla}(t)=\frac{f(t)-f(\rho(t))}{t-\rho(t)} .
$$

3. If $t$ is left-dense, then $f$ is nabla differentiable at $t$ if and only if the limit

$$
\lim _{s \rightarrow t} \frac{f(t)-f(s)}{t-s}
$$

exists as a finite number. In this case,

$$
f^{\nabla}(t)=\lim _{s \rightarrow t} \frac{f(t)-f(s)}{t-s} .
$$

4. If $f$ is nabla differentiable at $t$, then

$$
f^{\rho}(t)=f(t)-\nu(t) f^{\nabla}(t) .
$$

Remark 3. When $\mathbb{T}=\mathbb{R}$, then $f: \mathbb{R} \rightarrow \mathbb{R}$ is nabla differentiable at $t \in \mathbb{R}$ if and only if

$$
f^{\nabla}(t)=\lim _{s \rightarrow t} \frac{f(t)-f(s)}{t-s}
$$

exists, i.e., if and only if $f$ is differentiable at $t$ in the ordinary sense. When $\mathbb{T}=\mathbb{Z}$, then $f: \mathbb{Z} \rightarrow \mathbb{R}$ is always nabla differentiable at $t \in \mathbb{Z}$ and

$$
f^{\nabla}(t)=\frac{f(t)-f(\rho(t))}{t-\rho(t)}=f(t)-f(t-1)=: \nabla f(t),
$$

i.e., $\nabla$ is the usual backward difference operator defined by the last equation above. For any time scale $\mathbb{T}$, when $f$ is a constant, then $f^{\nabla}=0$; if $f(t)=k t$ for some constant $k$, then $f^{\nabla}=k$.

Theorem 8. (Theorem 8.41 in [14]) Suppose $f, g: \mathbb{T} \rightarrow$ $\mathbb{R}$ are nabla differentiable at $t \in \mathbb{T}_{\kappa}$. Then,

1. the sum $f+g: \mathbb{T} \rightarrow \mathbb{R}$ is nabla differentiable at $t$ and

$$
(f+g)^{\nabla}(t)=f^{\nabla}(t)+g^{\nabla}(t)
$$

2. for any constant $\alpha, \alpha f: \mathbb{T} \rightarrow \mathbb{R}$ is nabla differentiable at $t$ and

$$
(\alpha f)^{\nabla}(t)=\alpha f^{\nabla}(t)
$$

3. the product $f g: \mathbb{T} \rightarrow \mathbb{R}$ is nabla differentiable at $t$ and

$$
\begin{aligned}
(f g)^{\nabla}(t) & =f^{\nabla}(t) g(t)+f^{\rho}(t) g^{\nabla}(t) \\
& =f^{\nabla}(t) g^{\rho}(t)+f(t) g^{\nabla}(t) .
\end{aligned}
$$

Definition 9. A function $F: \mathbb{T} \rightarrow \mathbb{R}$ is called a nabla antiderivative of $f: \mathbb{T} \rightarrow \mathbb{R}$ provided $F^{\nabla}(t)=f(t)$ for all $t \in \mathbb{T}_{\kappa}$. In this case we define the nabla integral of $f$ from a to $b(a, b \in \mathbb{T})$ by

$$
\int_{a}^{b} f(t) \nabla t:=F(b)-F(a) .
$$

In order to exhibit a class of functions that possess a nabla antiderivative, the following definition is introduced. 
Definition 10. Let $\mathbb{T}$ be a time scale, $f: \mathbb{T} \rightarrow \mathbb{R}$. We say that function $f$ is ld-continuous if it is continuous at left-dense points and its right-sided limits exist (finite) at all right-dense points.

Theorem 11. (Theorem 8.45 in [14]) Every ldcontinuous function has a nabla antiderivative. In particular, if $a \in \mathbb{T}$, then the function $F$ defined by

$$
F(t)=\int_{a}^{t} f(\tau) \nabla \tau, \quad t \in \mathbb{T}
$$

is a nabla antiderivative of $f$.

The set of all ld-continuous functions $f: \mathbb{T} \rightarrow \mathbb{R}$ is denoted by $C_{\mathrm{ld}}^{0}=C_{\mathrm{ld}}=C_{\mathrm{ld}}(\mathbb{T})=C_{\mathrm{ld}}(\mathbb{T}, \mathbb{R})$, and the set of all nabla differentiable functions with ld-continuous derivative by $C_{\mathrm{ld}}^{1}=C_{\mathrm{ld}}^{1}(\mathbb{T}, \mathbb{R})$. In general, we say that $f \in C_{\mathrm{ld}}^{n}, n \in \mathbb{N}$, if $f^{\Delta} \in C_{\mathrm{ld}}^{n-1}$.

Theorem 12. (Theorems 8.46 and 8.47 in [14]) If $a, b$, $c \in \mathbb{T}, a \leq c \leq b, \alpha \in \mathbb{R}$, and $f, g \in C_{l d}(\mathbb{T}, \mathbb{R})$, then

1. $\int_{a}^{b}(f(t)+g(t)) \nabla t=\int_{a}^{b} f(t) \nabla t+\int_{a}^{b} g(t) \nabla t$

2. $\int_{a}^{b} \alpha f(t) \nabla t=\alpha \int_{a}^{b} f(t) \nabla t$

3. $\int_{a}^{b} f(t) \nabla t=-\int_{b}^{a} f(t) \nabla t$

4. $\int_{a}^{a} f(t) \nabla t=0$

5. $\int_{a}^{b} f(t) \nabla t=\int_{a}^{c} f(t) \nabla t+\int_{c}^{b} f(t) \nabla t$

6. If $f(t)>0$ for all $a<t \leq b$, then $\int_{a}^{b} f(t) \nabla t>0$;

7. If $t \in \mathbb{T}_{\kappa}$, then $\int_{\rho(t)}^{t} f(\tau) \nabla \tau=\nu(t) f(t)$.

Example 4. Let $a, b \in \mathbb{T}$ and $f \in C_{l d}(\mathbb{T}, \mathbb{R})$. For $\mathbb{T}=\mathbb{R}$, then $\int_{a}^{b} f(t) \nabla t=\int_{a}^{b} f(t) d t$, where the integral on the right side is the usual Riemann integral. For $\mathbb{T}=\mathbb{Z}$, then $\int_{a}^{b} f(t) \nabla t=\sum_{t=a+1}^{b} f(t)$ if $a<b, \int_{a}^{b} f(t) \nabla t=0$ if $a=b$, and $\int_{a}^{b} f(t) \nabla t=-\sum_{t=b+1}^{a} f(t)$ if $a>b$.

For more on the nabla calculus we refer the reader to [15, Chap. 3].

\subsection{Preliminaries to variational calculus}

Our goal is to obtain Euler-Lagrange type equations. Similar to the classical calculus of variations [23], integration by parts will play an important role. If functions $f, g: \mathbb{T} \rightarrow \mathbb{R}$ are delta and nabla differentiable with continuous derivatives, then the following formulas of integration by parts hold [14]:

$$
\begin{aligned}
\int_{a}^{b} f^{\sigma}(t) g^{\Delta}(t) \Delta t & =\left.(f g)(t)\right|_{t=a} ^{t=b}-\int_{a}^{b} f^{\Delta}(t) g(t) \Delta t, \\
\int_{a}^{b} f(t) g^{\Delta}(t) \Delta t & =\left.(f g)(t)\right|_{t=a} ^{t=b}-\int_{a}^{b} f^{\Delta}(t) g^{\sigma}(t) \Delta t, \\
\int_{a}^{b} f^{\rho}(t) g^{\nabla}(t) \nabla t & =\left.(f g)(t)\right|_{t=a} ^{t=b}-\int_{a}^{b} f^{\nabla}(t) g(t) \nabla t, \\
\int_{a}^{b} f(t) g^{\nabla}(t) \nabla t & =\left.(f g)(t)\right|_{t=a} ^{t=b}-\int_{a}^{b} f^{\nabla}(t) g^{\rho}(t) \nabla t
\end{aligned}
$$

The following fundamental lemma of the calculus of variations on time scales, involving a nabla derivative and a nabla integral, has been proved in [43].

Lemma 13. (The nabla Dubois-Reymond lemma [43, Lemma 14]). Let $f \in C_{l d}([a, b], \mathbb{R})$. If

$$
\int_{a}^{b} f(t) \eta^{\nabla}(t) \nabla t=0
$$

for all $\eta \in C_{l d}^{1}([a, b], \mathbb{R})$ with $\eta(a)=\eta(b)=0$, then $f(t)=c, t \in[a, b]_{\kappa}$, for some constant $c$.

Lemma 14 is the analogous delta version of Lemma 13.

Lemma 14. (The delta Dubois-Reymond lemma [12]). Let $g \in C_{r d}([a, b], \mathbb{R})$. If

$$
\int_{a}^{b} g(t) \eta^{\Delta}(t) \Delta t=0
$$

for all $\eta \in C_{r d}^{1}$ with $\eta(a)=\eta(b)=0$, then $g(t)=c$ on $[a, b]^{\kappa}$ for some $c \in \mathbb{R}$.

Proposition 15 gives a relationship between delta and nabla derivatives.

Proposition 15. (Theorems 2.5 and 2.6 of [5]). (i) If $f: \mathbb{T} \rightarrow \mathbb{R}$ is delta differentiable on $\mathbb{T}^{\kappa}$ and $f^{\Delta}$ is continuous on $\mathbb{T}^{\kappa}$, then $f$ is nabla differentiable on $\mathbb{T}_{\kappa}$ and

$$
f^{\nabla}(t)=\left(f^{\Delta}\right)^{\rho}(t) \quad \text { for all } t \in \mathbb{T}_{\kappa} .
$$

(ii) If $f: \mathbb{T} \rightarrow \mathbb{R}$ is nabla differentiable on $\mathbb{T}_{\kappa}$ and $f^{\nabla}$ is continuous on $\mathbb{T}_{\kappa}$, then $f$ is delta differentiable on $\mathbb{T}^{\kappa}$ and

$$
f^{\Delta}(t)=\left(f^{\nabla}\right)^{\sigma}(t) \quad \text { for all } t \in \mathbb{T}^{\kappa} .
$$

Remark 4. Note that, in general, $f^{\nabla}(t) \neq f^{\Delta}(\rho(t))$ and $f^{\Delta}(t) \neq f^{\nabla}(\sigma(t))$. In Proposition 15 the assumptions on the continuity of $f^{\Delta}$ and $f^{\nabla}$ are crucial. 
Proposition 16. ([5, Theorem 2.8]). Let $a, b \in \mathbb{T}$ with $a \leq b$ and let $f$ be a continuous function on $[a, b]$. Then,

$$
\begin{aligned}
& \int_{a}^{b} f(t) \Delta t=\int_{a}^{\rho(b)} f(t) \Delta t+(b-\rho(b)) f^{\rho}(b), \\
& \int_{a}^{b} f(t) \Delta t=(\sigma(a)-a) f(a)+\int_{\sigma(a)}^{b} f(t) \Delta t, \\
& \int_{a}^{b} f(t) \nabla t=\int_{a}^{\rho(b)} f(t) \nabla t+(b-\rho(b)) f(b), \\
& \int_{a}^{b} f(t) \nabla t=(\sigma(a)-a) f^{\sigma}(a)+\int_{\sigma(a)}^{b} f(t) \nabla t .
\end{aligned}
$$

We end our brief review of the calculus on time scales with a relationship between the delta and nabla integrals.

Proposition 17. ([27, Proposition 7]). If function $f$ : $\mathbb{T} \rightarrow \mathbb{R}$ is continuous, then for all $a, b \in \mathbb{T}$ with $a<b$ we have

$$
\begin{aligned}
& \int_{a}^{b} f(t) \Delta t=\int_{a}^{b} f^{\rho}(t) \nabla t \\
& \int_{a}^{b} f(t) \nabla t=\int_{a}^{b} f^{\sigma}(t) \Delta t
\end{aligned}
$$

\section{The $\Delta-\nabla$ calculus of variations}

We consider the problem of extremizing the variational functional (6) subject to given boundary conditions $y(a)=\alpha$ and $y(b)=\beta$ :

$$
\begin{gathered}
\mathcal{J}(y)=\left(\int_{a}^{b} L_{\Delta}[y](t) \Delta t\right)\left(\int_{a}^{b} L_{\nabla}\{y\}(t) \nabla t\right) \rightarrow \operatorname{extr} \\
y \in C_{\diamond}^{1}([a, b], \mathbb{R}) \\
y(a)=\alpha, \quad y(b)=\beta,
\end{gathered}
$$

where $C_{\diamond}^{1}([a, b], \mathbb{R})$ denote the class of functions $y$ : $[a, b] \rightarrow \mathbb{R}$ with $y^{\Delta}$ continuous on $[a, b]^{\kappa}$ and $y^{\nabla}$ continuous on $[a, b]_{\kappa}$.

Before presenting the Euler-Lagrange equations for problem (12) we introduce the definition of weak local extremum.

Definition 18. We say that $\hat{y} \in C_{\diamond}^{1}([a, b], \mathbb{R})$ is a weak local minimizer (respectively weak local maximizer) for problem (12) if there exists $\delta>0$ such that

$$
\mathcal{J}(\hat{y}) \leq \mathcal{J}(y) \quad \text { respectively } \mathcal{J}(\hat{y}) \geq \mathcal{J}(y))
$$

for all $y \in C_{\diamond}^{1}([a, b], \mathbb{R})$ satisfying the boundary conditions $y(a)=\alpha$ and $y(b)=\beta$, and

$$
\|y-\hat{y}\|_{1, \infty}<\delta
$$

where

$\|y\|_{1, \infty}:=\left\|y^{\sigma}\right\|_{\infty}+\left\|y^{\rho}\right\|_{\infty}+\left\|y^{\Delta}\right\|_{\infty}+\left\|y^{\nabla}\right\|_{\infty}$ and

$$
\|y\|_{\infty}:=\sup _{t \in[a, b]_{\kappa}^{\kappa}}|y(t)|
$$

\subsection{Euler-Lagrange equations}

Theorem 19 gives two different forms for the EulerLagrange equation on time scales associated with the variational problem (12).

Theorem 19. (The general Euler-Lagrange equations on time scales). If $\hat{y} \in C_{\diamond}^{1}$ is a weak local extremizer of problem (12), then $\hat{y}$ satisfies the following delta-nabla integral equations:

$$
\begin{gathered}
\mathcal{J}_{\nabla}(\hat{y})\left(\partial_{3} L_{\Delta}[\hat{y}](\rho(t))-\int_{a}^{\rho(t)} \partial_{2} L_{\Delta}[\hat{y}](\tau) \Delta \tau\right) \\
+\mathcal{J}_{\Delta}(\hat{y})\left(\partial_{3} L_{\nabla}\{\hat{y}\}(t)-\int_{a}^{t} \partial_{2} L_{\nabla}\{\hat{y}\}(\tau) \nabla \tau\right)=\text { const }
\end{gathered}
$$

for all $t \in[a, b]_{\kappa}$;

$$
\begin{array}{r}
\mathcal{J}_{\Delta}(\hat{y})\left(\partial_{3} L_{\nabla}\{\hat{y}\}(\sigma(t))-\int_{a}^{\sigma(t)} \partial_{2} L_{\nabla}\{\hat{y}\}(\tau) \nabla \tau\right) \\
+\mathcal{J}_{\nabla}(\hat{y})\left(\partial_{3} L_{\Delta}[\hat{y}](t)-\int_{a}^{t} \partial_{2} L_{\Delta}[\hat{y}](\tau) \Delta \tau\right)=\mathrm{const}
\end{array}
$$

for all $t \in[a, b]^{\kappa}$.

Remark 5. In the classical context (i.e., when $\mathbb{T}=\mathbb{R}$ ) the necessary conditions (13) and (14) coincide with the Euler-Lagrange equations (39) given in [18].

Proof. Suppose that $\mathcal{J}$ has a weak local extremum at $\hat{y}$. We consider the value of $\mathcal{J}$ at nearby functions $\hat{y}+\varepsilon \eta$, where $\varepsilon \in \mathbb{R}$ is a small parameter, $\eta \in C_{\diamond}^{1}([a, b], \mathbb{R})$ with $\eta(a)=\eta(b)=0$. Thus, function $\phi(\varepsilon)=\mathcal{J}(\hat{y}+\varepsilon \eta)$ has an extremum at $\varepsilon=0$. Using the first-order necessary optimality condition $\left.\phi^{\prime}(\varepsilon)\right|_{\varepsilon=0}=0$ we obtain:

$$
\begin{aligned}
0 & =\mathcal{J}_{\Delta}(\hat{y}) \int_{a}^{b}\left(\partial_{2} L_{\nabla}\{\hat{y}\}(t) \eta^{\rho}(t)+\partial_{3} L_{\nabla}\{\hat{y}\}(t) \eta^{\nabla}(t)\right) \nabla t \\
& +\mathcal{J}_{\nabla}(\hat{y}) \int_{a}^{b}\left(\partial_{2} L_{\Delta}[\hat{y}](t) \eta^{\sigma}(t)+\partial_{3} L_{\Delta}[\hat{y}](t) \eta^{\Delta}(t)\right) \Delta t .
\end{aligned}
$$

Let

$A(t)=\int_{a}^{t} \partial_{2} L_{\Delta}[\hat{y}](\tau) \Delta \tau, \quad B(t)=\int_{a}^{t} \partial_{2} L_{\nabla}\{\hat{y}\}(\tau) \nabla \tau$.

Then, $A^{\Delta}(t)=\partial_{2} L_{\Delta}[\hat{y}](t), B^{\nabla}(t)=\partial_{2} L_{\nabla}\{\hat{y}\}(t)$, and the first and third integration by parts formula in (7) tell us, respectively, that

$$
\begin{gathered}
\int_{a}^{b} \partial_{2} L_{\Delta}[\hat{y}](t) \eta^{\sigma}(t) \Delta t=\int_{a}^{b} A^{\Delta}(t) \eta^{\sigma}(t) \Delta t \\
=\left.A(t) \eta(t)\right|_{t=a} ^{t=b}-\int_{a}^{b} A(t) \eta^{\Delta}(t) \Delta t \\
=-\int_{a}^{b} A(t) \eta^{\Delta}(t) \Delta t
\end{gathered}
$$


and

$$
\begin{gathered}
\int_{a}^{b} \partial_{2} L_{\nabla}\{\hat{y}\}(t) \eta^{\rho}(t) \nabla t=\int_{a}^{b} B^{\nabla}(t) \eta^{\rho}(t) \nabla t \\
=\left.B(t) \eta(t)\right|_{t=a} ^{t=b}-\int_{a}^{b} B(t) \eta^{\nabla}(t) \nabla t \\
=-\int_{a}^{b} B(t) \eta^{\nabla}(t) \nabla t .
\end{gathered}
$$

If we denote $f(t)=\partial_{3} L_{\Delta}[\hat{y}](t)-A(t)$ and $g(t)=$ $\partial_{3} L_{\nabla}\{\hat{y}\}(t)-B(t)$, then we can write the necessary optimality condition (15) in the form

$\mathcal{J}_{\nabla}(\hat{y}) \int_{a}^{b} f(t) \eta^{\Delta}(t) \Delta t+\mathcal{J}_{\Delta}(\hat{y}) \int_{a}^{b} g(t) \eta^{\nabla}(t) \nabla t=0$

We now split the proof in two parts: (i) we prove (13) transforming the delta integral in (16) to a nabla integral by means of (10); (ii) we prove (14) transforming the nabla integral in (16) to a delta integral by means of (11). (i) By (10) the necessary optimality condition (16) is equivalent to

$$
\int_{a}^{b}\left(\mathcal{J}_{\nabla}(\hat{y}) f^{\rho}(t)\left(\eta^{\Delta}\right)^{\rho}(t)+\mathcal{J}_{\Delta}(\hat{y}) g(t) \eta^{\nabla}(t)\right) \nabla t=0
$$

and by (8) to

$$
\int_{a}^{b}\left(\mathcal{J}_{\nabla}(\hat{y}) f^{\rho}(t)+\mathcal{J}_{\Delta}(\hat{y}) g(t)\right) \eta^{\nabla}(t) \nabla t=0 .
$$

Applying Lemma 13 to (17) we prove (13):

$$
\mathcal{J}_{\nabla}(\hat{y}) f^{\rho}(t)+\mathcal{J}_{\Delta}(\hat{y}) g(t)=c \quad \forall t \in[a, b]_{\kappa},
$$

where $c$ is a constant. (ii) By (11) the necessary optimality condition (16) is equivalent to

$\int_{a}^{b}\left(\mathcal{J}_{\nabla}(\hat{y}) f(t) \eta^{\Delta}(t)+\mathcal{J}_{\Delta}(\hat{y}) g^{\sigma}(t)\left(\eta^{\nabla}\right)^{\sigma}(t)\right) \Delta t=0$ and by (9) to

$$
\int_{a}^{b}\left(\mathcal{J}_{\nabla}(\hat{y}) f(t)+\mathcal{J}_{\Delta}(\hat{y}) g^{\sigma}(t)\right) \eta^{\Delta}(t) \Delta t=0 .
$$

Applying Lemma 14 to (19) we prove (14):

$$
\mathcal{J}_{\nabla}(\hat{y}) f(t)+\mathcal{J}_{\Delta}(\hat{y}) g^{\sigma}(t)=c \quad \forall t \in[a, b]^{\kappa},
$$

where $c$ is a constant.

Corollary 20. Let $L_{\Delta}\left(t, y^{\sigma}, y^{\Delta}\right)=L_{\Delta}(t)$ and $\mathcal{J}_{\Delta}(\hat{y}) \neq 0$ (this is true, e.g., for $L_{\Delta} \equiv \frac{1}{b-a}$ for which $\mathcal{J}_{\Delta}=1$; cf. Remark 1). Then, $\partial_{2} L_{\Delta}=\partial_{3} L_{\Delta}=0$ and the Euler-Lagrange equation (13) takes the form

$$
\partial_{3} L_{\nabla}\{\hat{y}\}(t)-\int_{a}^{t} \partial_{2} L_{\nabla}\{\hat{y}\}(\tau) \nabla \tau=\text { const }
$$

for all $t \in[a, b]_{\kappa}$.
Remark 6. If $\hat{y} \in C_{l d}^{2}$, then nabla-differentiating (21) we obtain the Euler-Lagrange differential equation (4) as proved in [43]:

$$
\frac{\nabla}{\nabla t} \partial_{3} L_{\nabla}\{\hat{y}\}(t)-\partial_{2} L_{\nabla}\{\hat{y}\}(t)=0 \quad \forall t \in[a, b]_{\kappa^{2}} .
$$

Corollary 21. Let $L_{\nabla}\left(t, y^{\rho}, y^{\nabla}\right)=L_{\nabla}(t)$ and $\mathcal{J}_{\nabla}(\hat{y}) \neq 0$ (this is true, e.g., for $L_{\nabla} \equiv \frac{1}{b-a}$ for which $\mathcal{J}_{\nabla}=1$; cf. Remark 1). Then, $\partial_{2} L_{\nabla}=\partial_{3} L_{\nabla}=0$ and the Euler-Lagrange equation (14) takes the form

$$
\partial_{3} L_{\Delta}[\hat{y}](t)-\int_{a}^{t} \partial_{2} L_{\Delta}[\hat{y}](\tau) \Delta \tau=\text { const }
$$

for all $t \in[a, b]^{\kappa}$.

Remark 7. If $\hat{y} \in C_{r d}^{2}$, then delta-differentiating (22) we obtain the Euler-Lagrange differential equation (3) as proved in [12]:

$$
\frac{\Delta}{\Delta t} \partial_{3} L_{\Delta}[\hat{y}](t)-\partial_{2} L_{\Delta}[\hat{y}](t)=0 \quad \forall t \in[a, b]^{\kappa^{2}} .
$$

Example 5. Let $\mathbb{T}$ be a time scale with $0, \xi \in \mathbb{T}, 0<\xi$, and $(\mathbb{T} \backslash\{0, \xi\}) \cap[0, \xi] \neq \emptyset$. Consider the problem

$$
\begin{gathered}
\left.\mathcal{J}(y)=\left(\int_{0}^{\xi}\left(y^{\Delta}(t)\right)^{2} \Delta t\right)\left(\int_{0}^{\xi}\left(y^{\nabla}(t)\right)^{2}\right) \nabla t\right) \rightarrow \min \\
y(0)=0, \quad y(\xi)=\xi .
\end{gathered}
$$

Since

$$
L_{\Delta}=\left(y^{\Delta}\right)^{2}, \quad L_{\nabla}=\left(y^{\nabla}\right)^{2}
$$

we have

$$
\partial_{2} L_{\Delta}=0, \partial_{3} L_{\Delta}=2 y^{\Delta}, \partial_{2} L_{\nabla}=0, \partial_{3} L_{\nabla}=2 y^{\nabla} .
$$

Using equation (14) of Theorem 19 we get the following delta-nabla differential equation:

$$
2 A y^{\Delta}(t)+2 B y^{\nabla}(\sigma(t))=C,
$$

where $C \in \mathbb{R}$ and $A, B$ are the values of functionals $\mathcal{J}_{\nabla}$ and $\mathcal{J}_{\Delta}$ in a solution of problem (23), respectively. From (9) we can write equation (24) in the form

$$
2 A y^{\Delta}(t)+2 B y^{\Delta}=C .
$$

Observe that $A+B$ cannot be equal to 0 . Thus, solving equation (25) subject to the boundary conditions $y(0)=0$ and $y(\xi)=\xi$ we get $y(t)=t$ as a candidate local minimizer for the problem (23).

Example 6. Consider the problem

$$
\begin{gathered}
\mathcal{J}(y)=\left(\int_{0}^{1} t y^{\Delta}(t) \Delta t\right)\left(\int_{0}^{1}\left(y^{\nabla}(t)\right)^{2} \nabla t\right) \rightarrow \text { extr } \\
y(0)=0, \quad y(1)=1 .
\end{gathered}
$$

Since

$$
L_{\Delta}=t y^{\Delta}, \quad L_{\nabla}=\left(y^{\nabla}\right)^{2}
$$


we have

$\partial_{2} L_{\Delta}=0, \quad \partial_{3} L_{\Delta}=t, \quad \partial_{2} L_{\nabla}=0, \quad \partial_{3} L_{\nabla}=2 y^{\nabla}$.

Using equation (14) of Theorem 19 and relation (9), we get the following delta differential equation:

$$
A t+2 B y^{\Delta}(t)=C,
$$

where $C \in \mathbb{R}$ and $A, B$ are values of the functionals $\mathcal{J}_{\nabla}$ and $\mathcal{J}_{\Delta}$ in a solution of (26), respectively. Observe that $A \neq 0$, so that $B$ is also nonzero. A solution of (27) depends on the time scale. Let us solve, for example, this equation on $\mathbb{T}=\mathbb{R}$ and on $\mathbb{T}=\left\{0, \frac{1}{2}, 1\right\}$. On $\mathbb{T}=\mathbb{R}$ we obtain

$$
y(t)=-\frac{A}{4 B} t^{2}+\frac{4 B+A}{4 B} t .
$$

Substituting (28) into functionals $\mathcal{J}_{\nabla}$ and $\mathcal{J}_{\Delta}$ gives

$$
\left\{\begin{array}{l}
\frac{48 B^{2}+A^{2}}{48 B^{2}}=A \\
\frac{12 B-A}{24 B}=B .
\end{array}\right.
$$

Solving the system of equations (29) we obtain

$$
\left\{\begin{array} { l } 
{ A = 0 } \\
{ B = 0 , }
\end{array} \quad \left\{\begin{array}{l}
A=\frac{4}{3} \\
B=\frac{1}{3} .
\end{array}\right.\right.
$$

Therefore,

$$
y(t)=-t^{2}+2 t
$$

is a candidate extremizer for problem (26) on $\mathbb{T}=\mathbb{R}$. Note that nothing can be concluded from Theorem 19 as to whether y gives a minimum, a maximum, or neither of these, for $\mathcal{J}$.

The solution of (27) on $\mathbb{T}=\left\{0, \frac{1}{2}, 1\right\}$ is

$$
y(t)= \begin{cases}0 & \text { if } t=0 \\ \frac{1}{2}+\frac{A}{16 B} & \text { if } t=\frac{1}{2} \\ 1 & \text { if } t=1 .\end{cases}
$$

Constants $A$ and $B$ are determined by substituting (30) into functionals $\mathcal{J}_{\nabla}$ and $\mathcal{J}_{\Delta}$. The resulting system of equations is

$$
\left\{\begin{array}{l}
1+\frac{A^{2}}{64 B^{2}}=A \\
\frac{1}{4}-\frac{A}{32 B}=B .
\end{array}\right.
$$

Since system of equations (31) has no real solutions, we conclude that there exists no extremizer for problem (26) on $\mathbb{T}=\left\{0, \frac{1}{2}, 1\right\}$ among the set of functions that we consider to be admissible.

\subsection{Natural boundary conditions}

We consider now the situations when we want to minimize or maximize the variational functional $\mathcal{J}$ but $y(a)$ and/or $y(b)$ are free. Then,

$$
\left.A(t) \eta(t)\right|_{t=a} ^{t=b}=A(b) \eta(b)-A(a) \eta(a)=A(b) \eta(b)
$$

and

$$
\left.B(t) \eta(t)\right|_{t=a} ^{t=b}=B(b) \eta(b)-B(a) \eta(a)=B(b) \eta(b)
$$

do not vanish necessarily, and conditions (17) and (19) take, respectively, the form

$$
\begin{aligned}
& (A(b)+B(b)) \eta(b) \\
+ & \int_{a}^{b}\left(\mathcal{J}_{\nabla}(\hat{y}) f^{\rho}(t)+\mathcal{J}_{\Delta}(\hat{y}) g(t)\right) \eta^{\nabla}(t) \nabla t=0
\end{aligned}
$$

and

$$
\begin{aligned}
& (A(b)+B(b)) \eta(b) \\
& +\int_{a}^{b}\left(\mathcal{J}_{\nabla}(\hat{y}) f(t)+\mathcal{J}_{\Delta}(\hat{y}) g^{\sigma}(t)\right) \eta^{\Delta}(t) \Delta t=0 .
\end{aligned}
$$

Since (32) and (33) are valid for an arbitrary function $\eta$, in particular they hold for the subclass of functions $\eta$ for which $\eta(a)=\eta(b)=0$. Thus, the same Euler-Lagrange conditions (18) and (20) of Theorem 19 are obtained and both (32) and (33) simplify to

$$
(c+A(b)+B(b)) \eta(b)-c \eta(a)=0 .
$$

If $y(a)$ is free, $\eta(a)$ is arbitrary and we conclude that $c=0$; if $y(b)$ is free, $\eta(b)$ is arbitrary and we conclude that $c+A(b)+B(b)=0$. The value of the constant $c$ is obtained from (18) and (20). We remark that (18) does not hold in $a$ in the case $a$ is right-scattered; and (20) does not hold in $b$ if $b$ is left-scattered. By (20) one has $c=\mathcal{J}_{\nabla}(\hat{y}) f(a)+\mathcal{J}_{\Delta}(\hat{y}) g(\sigma(a))$, and by (18) $c=\mathcal{J}_{\nabla}(\hat{y}) f(\rho(b))+\mathcal{J}_{\Delta}(\hat{y}) g(b)$. We have just proved the following result:

Theorem 22. (The general natural boundary conditions on time scales). If $\hat{y}$ is a weak local extremizer of the variational functional (6), then the Euler-Lagrange equations (13) and (14) hold, together with the natural condition

$$
\begin{aligned}
\mathcal{J}_{\Delta}(\hat{y})\left(\partial_{3} L_{\nabla}\{\hat{y}\}(\sigma(a))\right. & \left.-\int_{a}^{\sigma(a)} \partial_{2} L_{\nabla}\{\hat{y}\}(\tau) \nabla \tau\right) \\
& +\mathcal{J}_{\nabla}(\hat{y}) \partial_{3} L_{\Delta}[\hat{y}](a)=0
\end{aligned}
$$

when $y(a)$ is free; together with the natural condition

$$
\begin{gathered}
\mathcal{J}_{\nabla}(\hat{y})\left(\partial_{3} L_{\Delta}[\hat{y}](\rho(b))-\int_{a}^{\rho(b)} \partial_{2} L_{\Delta}[\hat{y}](\tau) \Delta \tau\right) \\
+\int_{a}^{b} \partial_{2} L_{\Delta}[\hat{y}](\tau) \Delta \tau \\
+\mathcal{J}_{\Delta}(\hat{y})\left(\partial_{3} L_{\nabla}\{\hat{y}\}(b)-\int_{a}^{b} \partial_{2} L_{\nabla}\{\hat{y}\}(\tau) \nabla \tau\right) \\
+\int_{a}^{b} \partial_{2} L_{\nabla}\{\hat{y}\}(\tau) \nabla \tau=0
\end{gathered}
$$

when $y(b)$ is free. 
Using Proposition 16, the next corollary is obtained:

Corollary 23. (The delta natural boundary conditions). If $\hat{y}$ is a weak local extremizer of the delta variational functional (1), then the Euler-Lagrange equation (22) holds together with the natural condition

$$
\partial_{3} L_{\Delta}[\hat{y}](a)=0
$$

when $y(a)$ is free; together with the natural condition

$$
\partial_{3} L_{\Delta}[\hat{y}](\rho(b))+(b-\rho(b)) \partial_{2} L_{\Delta}[\hat{y}](\rho(b))=0
$$

when $y(b)$ is free.

Analogous nabla natural conditions are also trivially obtained from Theorem 22:

Corollary 24. (The nabla natural boundary conditions). If $\hat{y}$ is a weak local extremizer of the nabla variational functional (2), then the Euler-Lagrange equation (21) holds together with the natural condition

$$
\partial_{3} L_{\nabla}\{\hat{y}\}(\sigma(a))-\int_{a}^{\sigma(a)} \partial_{2} L_{\nabla}\{\hat{y}\}(\tau) \nabla \tau=0
$$

when $y(a)$ is free; together with the natural condition

$$
\partial_{3} L_{\nabla}\{\hat{y}\}(b)=0
$$

when $y(b)$ is free.

Remark 8. The natural boundary condition (37) can be written in the equivalent form

$$
\partial_{3} L_{\nabla}\{\hat{y}\}(\sigma(a))-(\sigma(a)-a) \partial_{2} L_{\nabla}\{\hat{y}\}(a)=0 .
$$

In the classical context of the calculus of variations, i.e., when $\mathbb{T}=\mathbb{R}$, both (35) and (37) reduce to $\partial_{3} L\left(a, y(a), y^{\prime}(a)\right)=0$ and both (36) and (38) reduce to $\partial_{3} L\left(b, y(b), y^{\prime}(b)\right)=0$, for some given Lagrangian $L$, which are the standard natural boundary conditions of the calculus of variations (cf., e.g., [23]). For the particular case $\mathbb{T}=\mathbb{R}$ we obtain from our Theorem 22 a result in [18] that generalizes the classical natural boundary conditions to functionals given by the product of two integrals:

Corollary 25. (cf. [18]). If $\hat{y}$ is a weak extremizer of the variational functional

$$
\begin{aligned}
\mathcal{I}(y)= & \mathcal{I}_{1}(y) \mathcal{I}_{2}(y) \\
= & \left(\int_{a}^{b} L_{1}\left(t, y(t), y^{\prime}(t)\right) d t\right) \\
& \cdot\left(\int_{a}^{b} L_{2}\left(t, y(t), y^{\prime}(t)\right) d t\right),
\end{aligned}
$$

then the Euler-Lagrange equation

$$
\begin{gathered}
\mathcal{I}_{2}(\hat{y})\left(\partial_{3} L_{1}\left(t, \hat{y}(t), \hat{y}^{\prime}(t)\right)-\int_{a}^{t} \partial_{2} L_{1}\left(t, \hat{y}(t), \hat{y}^{\prime}(t)\right) d t\right) \\
+\mathcal{I}_{1}(\hat{y})\left(\partial_{3} L_{2}\left(t, \hat{y}(t), \hat{y}^{\prime}(t)\right)-\int_{a}^{t} \partial_{2} L_{2}\left(t, \hat{y}(t), \hat{y}^{\prime}(t)\right) d t\right) \\
=\text { const }
\end{gathered}
$$

holds for all $t \in[a, b]$. Moreover, the natural condition

$$
\begin{aligned}
& \mathcal{I}_{2}(\hat{y}) \partial_{3} L_{1}\left(a, \hat{y}(a), \hat{y}^{\prime}(a)\right) \\
&+ \mathcal{I}_{1}(\hat{y}) \partial_{3} L_{2}\left(a, \hat{y}(a), \hat{y}^{\prime}(a)\right)=0
\end{aligned}
$$

holds when $y(a)$ is free; the natural condition

$$
\begin{gathered}
\mathcal{I}_{2}(\hat{y}) \partial_{3} L_{1}\left(b, \hat{y}(b), \hat{y}^{\prime}(b)\right)+\mathcal{I}_{1}(\hat{y}) \partial_{3} L_{2}\left(b, \hat{y}(b), \hat{y}^{\prime}(b)\right) \\
\quad+\left(1-\mathcal{I}_{2}(\hat{y})\right) \int_{a}^{b} \partial_{2} L_{1}\left(\tau, \hat{y}(\tau), \hat{y}^{\prime}(\tau)\right) d \tau \\
\quad+\left(1-\mathcal{I}_{1}(\hat{y})\right) \int_{a}^{b} \partial_{2} L_{2}\left(\tau, \hat{y}(\tau), \hat{y}^{\prime}(\tau)\right) d \tau=0
\end{gathered}
$$

holds when $y(b)$ is free.

Example 7. Let us consider the functional of Example 5 but where we are free to choose the value of $y$ at point $\xi$ :

$$
\begin{gathered}
\left.\mathcal{J}(y)=\left(\int_{0}^{\xi}\left(y^{\Delta}(t)\right)^{2} \Delta t\right)\left(\int_{0}^{\xi}\left(y^{\nabla}(t)\right)^{2}\right) \nabla t\right) \rightarrow \min \\
y(0)=0, \quad y(\xi) \text { free } .
\end{gathered}
$$

Solving the Euler-Lagrange equation (25) with $y(0)=0$ gives the extremal

$$
\hat{y}(t)=\frac{C}{2(A+B)} t .
$$

In this case the natural boundary condition (34) along (40) simplifies to

$$
A\left(\frac{C}{A+B}\right)+B\left(\frac{C}{A+B}\right)=0,
$$

that is, $C=0$ and the minimum is obtained by choosing $y(\xi)=0$. It is trivial to see that the extremal $\hat{y}(t) \equiv 0$ we just found is indeed the global minimizer: $\mathcal{J}(y) \geq 0$ for any function $y$, and $\mathcal{J}(\hat{y})=0$.

\subsection{The delta-nabla isoperimetric problem}

We consider now delta-nabla isoperimetric problems on time scales. The problem consists of extremizing

$\mathcal{L}(y)=\left(\int_{a}^{b} L_{\Delta}[y](t) \Delta t\right)\left(\int_{a}^{b} L_{\nabla}\{y\}(t) \nabla t\right) \longrightarrow \operatorname{extr}$

in the class of functions $y \in C_{\diamond}^{1}([a, b], \mathbb{R})$ satisfying the boundary conditions

$$
y(a)=\alpha, \quad y(b)=\beta,
$$

and the constraint

$$
\mathcal{K}(y)=\left(\int_{a}^{b} K_{\Delta}[y](t) \Delta t\right)\left(\int_{a}^{b} K_{\nabla}\{y\}(t) \nabla t\right)=k
$$

where $\alpha, \beta, k$ are given real numbers. 
Definition 26. We say that $\hat{y} \in C_{\diamond}^{1}([a, b], \mathbb{R})$ is a weak local minimizer (respectively weak local maximizer) for problem (41)-(43) if there exists $\delta>0$ such that

$$
\mathcal{L}(\hat{y}) \leq \mathcal{L}(y) \quad \text { respectively } \mathcal{L}(\hat{y}) \geq \mathcal{L}(y))
$$

for all $y \in C_{\diamond}^{1}([a, b], \mathbb{R})$ satisfying the boundary conditions (42), the constraint (43), and $\|y-\hat{y}\|_{1, \infty}<\delta$.

Definition 27. We say that $\hat{y} \in C_{\diamond}^{1}$ is an extremal for $\mathcal{K}$ if $\hat{y}$ satisfies the delta-nabla integral equations (13) and (14) for $\mathcal{K}$, i.e.,

$$
\begin{gathered}
\mathcal{K}_{\nabla}(\hat{y})\left(\partial_{3} K_{\Delta}[\hat{y}](\rho(t))-\int_{a}^{\rho(t)} \partial_{2} K_{\Delta}[\hat{y}](\tau) \Delta \tau\right) \\
+\mathcal{K}_{\Delta}(\hat{y})\left(\partial_{3} K_{\nabla}\{\hat{y}\}(t)-\int_{a}^{t} \partial_{2} K_{\nabla}\{\hat{y}\}(\tau) \nabla \tau\right)=\text { const }
\end{gathered}
$$

for all $t \in[a, b]_{\kappa}$;

$$
\begin{array}{r}
\mathcal{K}_{\Delta}(\hat{y})\left(\partial_{3} K_{\nabla}\{\hat{y}\}(\sigma(t))-\int_{a}^{\sigma(t)} \partial_{2} K_{\nabla}\{\hat{y}\}(\tau) \nabla \tau\right) \\
+\mathcal{K}_{\nabla}(\hat{y})\left(\partial_{3} K_{\Delta}[\hat{y}](t)-\int_{a}^{t} \partial_{2} K_{\Delta}[\hat{y}](\tau) \Delta \tau\right)=\mathrm{const}
\end{array}
$$

for all $t \in[a, b]^{\kappa}$. An extremizer (i.e., a weak local minimizer or a weak local maximizer) for the problem (41)(43) that is not an extremal for $\mathcal{K}$ is said to be a normal extremizer; otherwise (i.e., if it is an extremal for $\mathcal{K}$ ), the extremizer is said to be abnormal.

Theorem 28. If $\hat{y} \in C_{\diamond}^{1}([a, b], \mathbb{R})$ is a normal extremizer for the isoperimetric problem (41)-(43), then there exists $\lambda \in \mathbb{R}$ such that $\hat{y}$ satisfies the following delta-nabla integral equations:

$$
\begin{aligned}
& \mathcal{L}_{\nabla}(\hat{y})\left(\partial_{3} L_{\Delta}[\hat{y}](\rho(t))-\int_{a}^{\rho(t)} \partial_{2} L_{\Delta}[\hat{y}](\tau) \Delta \tau\right) \\
& +\mathcal{L}_{\Delta}(\hat{y})\left(\partial_{3} L_{\nabla}\{\hat{y}\}(t)-\int_{a}^{t} \partial_{2} L_{\nabla}\{\hat{y}\}(\tau) \nabla \tau\right) \\
& -\lambda\left\{\mathcal{K}_{\nabla}(\hat{y})\left(\partial_{3} K_{\Delta}[\hat{y}](\rho(t))-\int_{a}^{\rho(t)} \partial_{2} K_{\Delta}[\hat{y}](\tau) \Delta \tau\right)\right. \\
& \left.+\mathcal{K}_{\Delta}(\hat{y})\left(\partial_{3} K_{\nabla}\{\hat{y}\}(t)-\int_{a}^{t} \partial_{2} K_{\nabla}\{\hat{y}\}(\tau) \nabla \tau\right)\right\}=\text { const }
\end{aligned}
$$

for all $t \in[a, b]_{\kappa}$;

$$
\begin{aligned}
& \mathcal{L}_{\nabla}(\hat{y})\left(\partial_{3} L_{\Delta}[\hat{y}](t)-\int_{a}^{t} \partial_{2} L_{\Delta}[\hat{y}](\tau) \Delta \tau\right) \\
+ & \mathcal{L}_{\Delta}(\hat{y})\left(\partial_{3} L_{\nabla}\{\hat{y}\}(\sigma(t))-\int_{a}^{\sigma(t)} \partial_{2} L_{\nabla}\{\hat{y}\}(\tau) \nabla \tau\right) \\
- & \lambda\left\{\mathcal{K}_{\Delta}(\hat{y})\left(\partial_{3} K_{\nabla}\{\hat{y}\}(\sigma(t))-\int_{a}^{\sigma(t)} \partial_{2} K_{\nabla}\{\hat{y}\}(\tau) \nabla \tau\right)\right. \\
+ & \left.\mathcal{K}_{\nabla}(\hat{y})\left(\partial_{3} K_{\Delta}[\hat{y}](t)-\int_{a}^{t} \partial_{2} K_{\Delta}[\hat{y}](\tau) \Delta \tau\right)\right\}=\text { const }
\end{aligned}
$$

for all $t \in[a, b]^{\kappa}$.

Proof.Consider a variation of $\hat{y}$, say $\bar{y}=\hat{y}+\varepsilon_{1} \eta_{1}+$ $\varepsilon_{2} \eta_{2}$, where for each $i \in\{1,2\}, \eta_{i} \in C_{\diamond}^{1}([a, b], \mathbb{R})$ and $\eta_{i}(a)=\eta_{i}(b)=0$, and $\varepsilon_{i}$ is a sufficiently small parameter $\left(\varepsilon_{1}\right.$ and $\varepsilon_{2}$ must be such that $\|\bar{y}-\hat{y}\|_{1, \infty}<\delta$ for some $\delta>0)$. Here, $\eta_{1}$ is an arbitrary fixed function and $\eta_{2}$ is a fixed function that will be chosen later. Define the real function

$$
\begin{aligned}
\bar{K} & \left(\varepsilon_{1}, \varepsilon_{2}\right)=\mathcal{K}(\bar{y}) \\
& =\left(\int_{a}^{b} K_{\Delta}[\bar{y}](t) \Delta t\right)\left(\int_{a}^{b} K_{\nabla}\{\bar{y}\}(t) \nabla t\right)-k .
\end{aligned}
$$

We have $\left.\frac{\partial \bar{K}}{\partial \varepsilon_{2}}\right|_{(0,0)}=0$, that is,

$$
\begin{gathered}
0=\mathcal{K}_{\nabla}(\hat{y}) \int_{a}^{b}\left(\partial_{2} K_{\Delta}[\hat{y}](t) \eta_{2}^{\sigma}(t)+\partial_{3} K_{\Delta}[\hat{y}](t) \eta_{2}^{\Delta}(t)\right) \Delta t \\
+\mathcal{K}_{\Delta}(\hat{y}) \int_{a}^{b}\left(\partial_{2} K_{\nabla}\{\hat{y}\}(t) \eta_{2}^{\rho}(t)+\partial_{3} K_{\nabla}\{\hat{y}\}(t) \eta_{2}^{\nabla}(t)\right) \nabla t .
\end{gathered}
$$

Since $\eta_{2}(a)=\eta_{2}(b)=0$, the first and third integration by parts formula in (7) give

$$
\begin{gathered}
\int_{a}^{b} \partial_{2} K_{\Delta}[\hat{y}](t) \eta_{2}^{\sigma}(t) \Delta t=\left.\int_{a}^{t} \partial_{2} K_{\Delta}[\hat{y}](\tau) \Delta \tau \eta_{2}(t)\right|_{t=a} ^{t=b} \\
-\int_{a}^{b}\left(\int_{a}^{t} \partial_{2} K_{\Delta}[\hat{y}](\tau) \Delta \tau\right) \eta_{2}^{\Delta}(t) \Delta t \\
=-\int_{a}^{b}\left(\int_{a}^{t} \partial_{2} K_{\Delta}[\hat{y}](\tau) \Delta \tau\right) \eta_{2}^{\Delta}(t) \Delta t
\end{gathered}
$$

and

$$
\begin{gathered}
\int_{a}^{b} \partial_{2} K_{\nabla}\{\hat{y}\}(t) \eta_{2}^{\rho}(t) \nabla t=\left.\int_{a}^{t} \partial_{2} K_{\nabla}\{\hat{y}\}(\tau) \nabla \tau \eta_{2}(t)\right|_{t=a} ^{t=b} \\
-\int_{a}^{b}\left(\int_{a}^{t} \partial_{2} K_{\nabla}\{\hat{y}\}(\tau) \nabla \tau\right) \eta_{2}^{\nabla}(t) \nabla t \\
=-\int_{a}^{b}\left(\int_{a}^{t} \partial_{2} K_{\nabla}\{\hat{y}\}(\tau) \nabla \tau\right) \eta_{2}^{\nabla}(t) \nabla t
\end{gathered}
$$


Therefore,

$$
\begin{aligned}
\left.\frac{\partial \bar{K}}{\partial \varepsilon_{2}}\right|_{(0,0)} & =\mathcal{K}_{\nabla}(\hat{y}) \int_{a}^{b}\left(\partial_{3} K_{\Delta}[\hat{y}](t)\right. \\
& \left.-\int_{a}^{t} \partial_{2} K_{\Delta}[\hat{y}](\tau) \Delta \tau\right) \eta_{2}^{\Delta}(t) \Delta t \\
& +\mathcal{K}_{\Delta}(\hat{y}) \int_{a}^{b}\left(\partial_{3} K_{\nabla}\{\hat{y}\}(t)\right. \\
& \left.-\int_{a}^{t} \partial_{2} K_{\nabla}\{\hat{y}\}(\tau) \nabla \tau\right) \eta_{2}^{\nabla}(t) \nabla t
\end{aligned}
$$

Let

$$
f(t)=\mathcal{K}_{\nabla}(\hat{y})\left(\partial_{3} K_{\Delta}[\hat{y}](t)-\int_{a}^{t} \partial_{2} K_{\Delta}[\hat{y}](\tau) \Delta \tau\right)
$$

and

$$
g(t)=\mathcal{K}_{\Delta}(\hat{y})\left(\partial_{3} K_{\nabla}\{\hat{y}\}(t)-\int_{a}^{t} \partial_{2} K_{\nabla}\{\hat{y}\}(\tau) \nabla \tau\right) .
$$

We can then write equation (48) in the form

$$
\left.\frac{\partial \bar{K}}{\partial \varepsilon_{2}}\right|_{(0,0)}=\int_{a}^{b} f(t) \eta_{2}^{\Delta}(t) \Delta t+\int_{a}^{b} g(t) \eta_{2}^{\nabla}(t) \nabla t .
$$

Transforming the delta integral in (49) to a nabla integral by means of (10) we obtain

$$
\left.\frac{\partial \bar{K}}{\partial \varepsilon_{2}}\right|_{(0,0)}=\int_{a}^{b} f^{\rho}(t)\left(\eta_{2}^{\Delta}\right)^{\rho}(t) \nabla t+\int_{a}^{b} g(t) \eta_{2}^{\nabla}(t) \nabla t
$$

and by (8)

$$
\left.\frac{\partial \bar{K}}{\partial \varepsilon_{2}}\right|_{(0,0)}=\int_{a}^{b}\left(f^{\rho}(t)+g(t)\right) \eta_{2}^{\nabla}(t) \nabla t .
$$

As $\hat{y}$ is a normal extremizer we conclude, by Lemma 13 and equation (45), that there exists $\eta_{2}$ such that $\left.\frac{\partial \bar{K}}{\partial \varepsilon_{2}}\right|_{(0,0)} \neq 0$. Note that the same result can be obtained by transforming the nabla integral in (49) to a delta integral by means of (11) and then using Lemma 14 and equation (44). Since $\bar{K}(0,0)=0$, by the implicit function theorem we conclude that there exists a function $\varepsilon_{2}$ defined in the neighborhood of zero, such that $\bar{K}\left(\varepsilon_{1}, \varepsilon_{2}\left(\varepsilon_{1}\right)\right)=0$, i.e., we may choose a subset of variations $\bar{y}$ satisfying the isoperimetric constraint.

Let us now consider the real function

$$
\begin{aligned}
\bar{L}\left(\varepsilon_{1}, \varepsilon_{2}\right) & =\mathcal{L}(\bar{y}) \\
= & \left(\int_{a}^{b} L_{\Delta}[\bar{y}](t) \Delta t\right)\left(\int_{a}^{b} L_{\nabla}\{\bar{y}\}(t) \nabla t\right) .
\end{aligned}
$$

By hypothesis, $(0,0)$ is an extremal of $\bar{L}$ subject to the constraint $\bar{K}=0$ and $\nabla \bar{K}(0,0) \neq \mathbf{0}$. By the Lagrange multiplier rule, there exists some real $\lambda$ such that $\nabla(\bar{L}(0,0)-\lambda \bar{K}(0,0))=\mathbf{0}$. Having in mind that $\eta_{1}(a)=\eta_{1}(b)=0$, we can write

$$
\begin{aligned}
\left.\frac{\partial \bar{L}}{\partial \varepsilon_{1}}\right|_{(0,0)}= & \mathcal{L}_{\nabla}(\hat{y}) \int_{a}^{b}\left(\partial_{3} L_{\Delta}[\hat{y}](t)\right. \\
- & \left.\int_{a}^{t} \partial_{2} L_{\Delta}[\hat{y}](\tau) \Delta \tau\right) \eta_{1}^{\Delta}(t) \Delta t \\
& +\mathcal{L}_{\Delta}(\hat{y}) \int_{a}^{b}\left(\partial_{3} L_{\nabla}\{\hat{y}\}(t)\right. \\
& \left.-\int_{a}^{t} \partial_{2} L_{\nabla}\{\hat{y}\}(\tau) \nabla \tau\right) \eta_{1}^{\nabla}(t) \nabla t
\end{aligned}
$$

and

$$
\begin{aligned}
\left.\frac{\partial \bar{K}}{\partial \varepsilon_{1}}\right|_{(0,0)}= & \mathcal{K}_{\nabla}(\hat{y}) \int_{a}^{b}\left(\partial_{3} K_{\Delta}[\hat{y}](t)\right. \\
- & \left.\int_{a}^{t} \partial_{2} K_{\Delta}[\hat{y}](\tau) \Delta \tau\right) \eta_{1}^{\Delta}(t) \Delta t \\
& +\mathcal{K}_{\Delta}(\hat{y}) \int_{a}^{b}\left(\partial_{3} K_{\nabla}\{\hat{y}\}(t)\right. \\
& \left.-\int_{a}^{t} \partial_{2} K_{\nabla}\{\hat{y}\}(\tau) \nabla \tau\right) \eta_{1}^{\nabla}(t) \nabla t .
\end{aligned}
$$

Let

$$
m(t)=\mathcal{L}_{\nabla}(\hat{y})\left(\partial_{3} L_{\Delta}[\hat{y}](t)-\int_{a}^{t} \partial_{2} L_{\Delta}[\hat{y}](\tau) \Delta \tau\right)
$$

and

$$
n(t)=\mathcal{L}_{\Delta}(\hat{y})\left(\partial_{3} L_{\nabla}\{\hat{y}\}(t)-\int_{a}^{t} \partial_{2} L_{\nabla}\{\hat{y}\}(\tau) \nabla \tau\right) .
$$

Then equations (50) and (51) can be written in the form

$$
\left.\frac{\partial \bar{L}}{\partial \varepsilon_{1}}\right|_{(0,0)}=\int_{a}^{b} m(t) \eta_{1}^{\Delta}(t) \Delta t+\int_{a}^{b} n(t) \eta_{1}^{\nabla}(t) \nabla t
$$

and

$$
\left.\frac{\partial \bar{K}}{\partial \varepsilon_{1}}\right|_{(0,0)}=\int_{a}^{b} f(t) \eta_{1}^{\Delta}(t) \Delta t+\int_{a}^{b} g(t) \eta_{1}^{\nabla}(t) \nabla t .
$$

Transforming the delta integrals in the above equalities to nabla integrals by means of (10) and using (8) we obtain

$$
\left.\frac{\partial \bar{L}}{\partial \varepsilon_{1}}\right|_{(0,0)}=\int_{a}^{b}\left(m^{\rho}(t)+n(t)\right) \eta_{1}^{\nabla}(t) \nabla t
$$

and

$$
\left.\frac{\partial \bar{K}}{\partial \varepsilon_{1}}\right|_{(0,0)}=\int_{a}^{b}\left(f(t)^{\rho}+g(t)\right) \eta_{1}^{\nabla}(t) \nabla t .
$$


Therefore,

$$
\int_{a}^{b} \eta_{1}^{\Delta}(t)\left\{m^{\rho}(t)+n(t)-\lambda\left(f(t)^{\rho}+g(t)\right)\right\} \nabla t=0 .
$$

Since (52) holds for any $\eta_{1}$, by Lemma 13 we have

$$
m^{\rho}(t)+n(t)-\lambda\left(f(t)^{\rho}+g(t)\right)=c
$$

for some $c \in \mathbb{R}$ and all $t \in[a, b]_{\kappa}$. Hence, condition (46) holds. Equation (46) can also be obtained by transforming nabla integrals to delta integrals by means of (11) and then using Lemma 14 and equation (44).

In the particular case $L_{\nabla} \equiv \frac{1}{b-a}$ we get from Theorem 28 the main result of [22]:

Corollary 29 (Theorem 3.4 of [22]). Suppose that

$$
J(y)=\int_{a}^{b} L\left(t, y^{\sigma}(t), y^{\Delta}(t)\right) \Delta t
$$

has a local minimum at $y_{*}$ subject to the boundary conditions $y(a)=y_{a}$ and $y(b)=y_{b}$ and the isoperimetric constraint

$$
I(y)=\int_{a}^{b} g\left(t, y^{\sigma}(t), y^{\Delta}(t)\right) \Delta t=k .
$$

Assume that $y_{*}$ is not an extremal for the functional $I$. Then, there exists a Lagrange multiplier constant $\lambda$ such that $y_{*}$ satisfies the following equation:

$$
\partial_{3} F^{\Delta}\left(t, y_{*}^{\sigma}(t), y_{*}^{\Delta}(t)\right)-\partial_{2} F\left(t, y_{*}^{\sigma}(t), y_{*}^{\Delta}(t)\right)=0
$$

for all $t \in[a, b]^{\kappa^{2}}$, where $F=L-\lambda g$ and $\partial_{3} F^{\Delta}$ denotes the delta derivative of a composition.

One can easily cover abnormal extremizers within our result by introducing an extra multiplier $\lambda_{0}$.

Theorem 30. If $\hat{y} \in C_{\diamond}^{1}$ is an extremizer for the isoperimetric problem (41)-(43), then there exist two constants $\lambda_{0}$ and $\lambda$, not both zero, such that $\hat{y}$ satisfies the following delta-nabla integral equations:

$$
\begin{aligned}
& \lambda_{0}\left\{\mathcal{L}_{\nabla}(\hat{y})\left(\partial_{3} L_{\Delta}[\hat{y}](\rho(t))-\int_{a}^{\rho(t)} \partial_{2} L_{\Delta}[\hat{y}](\tau) \Delta \tau\right)\right. \\
& \left.+\mathcal{L}_{\Delta}(\hat{y})\left(\partial_{3} L_{\nabla}\{\hat{y}\}(t)-\int_{a}^{t} \partial_{2} L_{\nabla}\{\hat{y}\}(\tau) \nabla \tau\right)\right\} \\
& -\lambda\left\{\mathcal{K}_{\nabla}(\hat{y})\left(\partial_{3} K_{\Delta}[\hat{y}](\rho(t))-\int_{a}^{\rho(t)} \partial_{2} K_{\Delta}[\hat{y}](\tau) \Delta \tau\right)\right. \\
& \left.+\mathcal{K}_{\Delta}(\hat{y})\left(\partial_{3} K_{\nabla}\{\hat{y}\}(t)-\int_{a}^{t} \partial_{2} K_{\nabla}\{\hat{y}\}(\tau) \nabla \tau\right)\right\}=\text { const }
\end{aligned}
$$

for all $t \in[a, b]_{\kappa}$;

$$
\begin{aligned}
& \lambda_{0}\left\{\mathcal{L}_{\nabla}(\hat{y})\left(\partial_{3} L_{\Delta}[\hat{y}](t)-\int_{a}^{t} \partial_{2} L_{\Delta}[\hat{y}](\tau) \Delta \tau\right)\right. \\
+ & \left.\mathcal{L}_{\Delta}(\hat{y})\left(\partial_{3} L_{\nabla}\{\hat{y}\}(\sigma(t))-\int_{a}^{\sigma(t)} \partial_{2} L_{\nabla}\{\hat{y}\}(\tau) \nabla \tau\right)\right\} \\
- & \lambda\left\{\mathcal{K}_{\Delta}(\hat{y})\left(\partial_{3} K_{\nabla}\{\hat{y}\}(\sigma(t))-\int_{a}^{\sigma(t)} \partial_{2} K_{\nabla}\{\hat{y}\}(\tau) \nabla \tau\right)\right. \\
+ & \left.\mathcal{K}_{\nabla}(\hat{y})\left(\partial_{3} K_{\Delta}[\hat{y}](t)-\int_{a}^{t} \partial_{2} K_{\Delta}[\hat{y}](\tau) \Delta \tau\right)\right\}=\text { const }
\end{aligned}
$$

for all $t \in[a, b]^{\kappa}$.

Proof.Following the proof of Theorem 28, since $(0,0)$ is an extremal of $\bar{L}$ subject to the constraint $\bar{K}=0$, the extended Lagrange multiplier rule (see for instance [47, Theorem 4.1.3]) asserts the existence of reals $\lambda_{0}$ and $\lambda$, not both zero, such that $\nabla\left(\lambda_{0} \bar{L}(0,0)-\lambda \bar{K}(0,0)\right)=\mathbf{0}$. Therefore,

$\int_{a}^{b} \eta_{1}^{\Delta}(t)\left\{\lambda_{0}\left(m^{\rho}(t)+n(t)\right)-\lambda\left(f(t)^{\rho}+g(t)\right)\right\} \nabla t=0$.

Since (55) holds for any $\eta_{1}$, by Lemma 13, we have

$$
\lambda_{0}\left(m^{\rho}(t)+n(t)\right)-\lambda\left(f(t)^{\rho}+g(t)\right)=c
$$

for some $c \in \mathbb{R}$ and all $t \in[a, b]_{\kappa}$. This establishes equation (53). Equation (54) can be shown using a similar technique.

Remark 9. If $\hat{y} \in C_{\diamond}^{1}$ is an extremizer for the isoperimetric problem (41)-(43), then we can choose $\lambda_{0}=1$ in Theorem 30 and obtain Theorem 28. For abnormal extremizers, Theorem 30 holds with $\lambda_{0}=0$. The condition $\left(\lambda_{0}, \lambda\right) \neq \mathbf{0}$ guarantees that Theorem 30 is a useful necessary optimality condition.

In the particular case $L_{\Delta} \equiv \frac{1}{b-a}$ we get from Theorem 30 a result of [3]:

Corollary 31 (Theorem 2 of [3]). If y is a local minimizer or maximizer for

$$
I[y]=\int_{a}^{b} f\left(t, y^{\rho}(t), y^{\nabla}(t)\right) \nabla t
$$

subject to the boundary conditions $y(a)=\alpha$ and $y(b)=$ $\beta$ and the nabla-integral constraint

$$
J[y]=\int_{a}^{b} g\left(t, y^{\rho}(t), y^{\nabla}(t)\right) \nabla t=\Lambda,
$$

then there exist two constants $\lambda_{0}$ and $\lambda$, not both zero, such that

$$
\partial_{3} K^{\nabla}\left(t, y^{\rho}(t), y^{\nabla}(t)\right)-\partial_{2} K\left(t, y^{\rho}(t), y^{\nabla}(t)\right)=0
$$

(53) for all $t \in[a, b]_{\kappa}$, where $K=\lambda_{0} f-\lambda g$. 
Example 8. Let $\mathbb{T}=\{1,2,3, \ldots, M\}$, where $M \in \mathbb{N}$ and $M \geq 2$. Consider the problem

$$
\begin{gathered}
\left.\left(\int_{0}^{M}\left(y^{\Delta}(t)\right)^{2} \Delta t\right)\left(\int_{0}^{M}\left(y^{\nabla}(t)\right)^{2}+y^{\nabla}(t)\right) \nabla t\right) \rightarrow \min \\
y(0)=0, \quad y(M)=M,
\end{gathered}
$$

subject to the constraint

$$
\mathcal{K}(y)=\int_{0}^{M} t y^{\Delta}(t) \Delta t=1
$$

Since

$$
\begin{aligned}
& L_{\Delta}=\left(y^{\Delta}\right)^{2}, \quad L_{\nabla}=\left(y^{\nabla}\right)^{2}+y^{\nabla}, \\
& K_{\Delta}=t y^{\Delta}, \quad K_{\nabla}=\frac{1}{M}
\end{aligned}
$$

we have

$\partial_{2} L_{\Delta}=0, \partial_{3} L_{\Delta}=2 y^{\Delta}, \partial_{2} L_{\nabla}=0, \partial_{3} L_{\nabla}=2 y^{\nabla}+1$,

and

$$
\partial_{2} K_{\Delta}=0, \quad \partial_{3} K_{\Delta}=t, \quad \partial_{2} K_{\nabla}=0, \quad \partial_{3} K_{\nabla}=0 .
$$

As

$$
\begin{aligned}
& \mathcal{K}_{\Delta}(\hat{y})\left(\partial_{3} K_{\nabla}\{\hat{y}\}(\sigma(t))-\int_{a}^{\sigma(t)} \partial_{2} K_{\nabla}\{\hat{y}\}(\tau) \nabla \tau\right) \\
& +\mathcal{K}_{\nabla}(\hat{y})\left(\partial_{3} K_{\Delta}[\hat{y}](t)-\int_{a}^{t} \partial_{2} K_{\Delta}[\hat{y}](\tau) \Delta \tau\right)=t
\end{aligned}
$$

there are no abnormal extremals for the problem (56)(57). Applying equation (47) of Theorem 28 we get the following delta-nabla differential equation:

$$
2 A y^{\Delta}(t)+B+2 B y^{\nabla}(\sigma(t))-\lambda t=C,
$$

where $C \in \mathbb{R}$ and $A, B$ are the values of functionals $\mathcal{L}_{\nabla}$ and $\mathcal{L}_{\Delta}$ in a solution of (56)-(57), respectively. Since $y^{\nabla}(\sigma(t))=y^{\Delta}(t)$, we can write equation (58) in the form

$$
2 A y^{\Delta}(t)+B+2 B y^{\Delta}-\lambda t=C .
$$

Observe that $B \neq 0$ and $A>2$. Hence, solving equation (59) subject to the boundary conditions $y(0)=0$ and $y(M)=M$ we get

$$
y(t)=\left[1-\frac{\lambda(M-t)}{4(A+B)}\right] t .
$$

Substituting (60) into (57) we obtain $\lambda=-\frac{(A+B)(M-2)}{12 M(M-1)}$. Hence,

$$
y(t)=\frac{\left(4 M^{2}-7 M-3 M t+6 t\right) t}{M(M-1)}
$$

is a candidate local minimizer for the problem (56)-(57).

\section{Conclusion}

The calculus of variations on time scales is an important subject under strong current research (see [10, 13, 19, 35, $36,41]$ and references therein). Here we review a general necessary optimality condition for problems of the calculus of variations on time scales [37, 38]. The proposed calculus of variations extends the problems with delta derivatives considered in $[12,22]$ and analogous nabla problems $[3,43]$ to more general cases described by the product of a delta and a nabla integral. Minimization of functionals given by the product of two integrals were considered by Euler himself, and are now receiving an increasing interest because of their nonlocal properties and their applications in economics [18]. We proved Euler-Lagrange type conditions for the generalized calculus of variations and corresponding natural boundary conditions.

The calculus of variations here promoted can be further developed. For instance, we can continue our study by proving sufficient optimality conditions. Moreover, the results here presented can be generalized in different ways: (i) to variational problems involving higher-order delta and nabla derivatives, unifying and extending the higher-order results on time scales of [21] and [43]; (ii) to problems of the calculus of variations on time scales introduced in [40], with a functional which is the composition of a certain scalar function $H$ with the delta integral of a vector valued field $f_{\Delta}$ and a nabla integral of a vector field $f_{\nabla}$, i.e., of the form

$H\left(\int_{a}^{b} f_{\Delta}\left(t, y^{\sigma}(t), y^{\Delta}(t)\right) \Delta t, \int_{a}^{b} f_{\nabla}\left(t, y^{\rho}(t), y^{\nabla}(t)\right) \nabla t\right)$.

Euler-Lagrange equations and natural boundary conditions for such problems on time scales can be proved, and present results obtained as corollaries.

\section{Acknowledgements}

This work is supported by the Portuguese Foundation for Science and Technology (FCT) through the Center for Research and Development in Mathematics and Applications (CIDMA).

\section{References}

1. R. Agarwal, M. Bohner, D. O'Regan, A. Peterson, Dynamic equations on time scales: a survey, J. Comput. Appl. Math. 141(1-2), 1-26, (2002).

2. C.D. Ahlbrandt, C. Morian, Partial differential equations on time scales, J. Comput. Appl. Math. 141(12), 35-55, (2002).

3. R. Almeida, D.F.M. Torres, Isoperimetric problems on time scales with nabla derivatives, J. Vib. Control 15(6), 951-958, (2009). 
4. F.M. Atici, D.C. Biles, A. Lebedinsky, An application of time scales to economics, Math. Comput. Modelling 43(7-8), 718-726, (2006).

5. F.M. Atici, G.Sh. Guseinov, On Green's functions and positive solutions for boundary value problems on time scales, J. Comput. Appl. Math. 141(1-2), 7599, (2002).

6. F.M. Atici, C.S. McMahan, A comparison in the theory of calculus of variations on time scales with an application to the Ramsey model, Nonlinear Dyn. Syst. Theory 9(1), 1-10, (2009).

7. F.M. Atici, F. Uysal, A production-inventory model of HMMS on time scales, Appl. Math. Lett. 21(3), 236-243, (2008).

8. B. Aulbach, S. Hilger, A unified approach to continuous and discrete dynamics. In Qualitative theory of differential equations, Szeged 1988, Colloq. Math. Soc. János Bolyai, 53 North-Holland, Amsterdam, 37-56, (1990).

9. G. Bangerezako, Variational q-calculus, J. Math. Anal. Appl. 289(2), 650-665, (2004).

10. Z. Bartosiewicz, N. Martins, D.F.M. Torres, The second Euler-Lagrange equation of variational calculus on time scales, Eur. J. Control 17(1), 1-10, (2011).

11. Z. Bartosiewicz, D.F.M. Torres, Noether's theorem on time scales, J. Math. Anal. Appl. 342(2), 12201226, (2008).

12. M. Bohner, Calculus of variations on time scales, Dynam. Systems Appl. 13(3-4), 339-349, (2004).

13. M. Bohner, R.A.C. Ferreira, D.F.M. Torres, Integral inequalities and their applications to the calculus of variations on time scales, Math. Inequal. Appl. 13(3), 511-522, (2010).

14. M. Bohner, A. Peterson, Dynamic equations on time scales, Birkhäuser Boston, Boston, MA, (2001).

15. M. Bohner, A. Peterson, Advances in dynamic equations on time scales, Birkhäuser Boston, Inc., Boston, MA, (2003).

16. A.M.C. Brito da Cruz, N. Martins, D.F.M. Torres, Higher-order Hahn's quantum variational calculus, Nonlinear Anal. (in press). DOI: 10.1016/j.na.2011.01.015.

17. M.C. Caputo, Time scales: from nabla calculus to delta calculus and viceversa via duality, Int. J. Difference Equ. 5(1), 25-40, (2010).

18. E. Castillo, A. Luceño, P. Pedregal, Composition functionals in calculus of variations. Application to products and quotients, Math. Models Methods Appl. Sci. 18(1), 47-75, (2008).
19. R.A.C. Ferreira, A.B. Malinowska, D.F.M. Torres, Optimality conditions for the calculus of variations with higher-order delta derivatives, Appl. Math. Lett. 24(1), 87-92, (2011).

20. R.A.C. Ferreira, D.F.M. Torres, Remarks on the calculus of variations on time scales, Int. J. Ecol. Econ. Stat. 9(F07), 65-73, (2007).

21. R.A.C. Ferreira, D.F.M. Torres, Higher-order calculus of variations on time scales. In Mathematical control theory and finance, Springer, Berlin, 149159, (2008).

22. R.A.C. Ferreira, D.F.M. Torres, Isoperimetric problems of the calculus of variations on time scales. In Nonlinear Analysis and Optimization II, Contemporary Mathematics, 514, Amer. Math. Soc., Providence, RI, 123-131, (2010).

23. I.M. Gelfand, S.V. Fomin, Calculus of variations , Revised English edition translated and edited by Richard A. Silverman Prentice-Hall, Inc., Englewood Cliffs, N.J., (1963).

24. E. Girejko, A.B. Malinowska, D.F.M. Torres, A unified approach to the calculus of variations on time scales, Proceedings of 2010 CCDC, Xuzhou, China, May 26-28, 2010. In: IEEE Catalog Number CFP1051D-CDR, 595-600, (2010). DOI: 10.1109/CCDC.2010.5498972.

25. E. Girejko, A.B. Malinowska, D.F.M. Torres, Deltanabla optimal control problems, J. Vib. Control (in press). DOI: 10.1177/1077546310381271.

26. E. Girejko, A.B. Malinowska, D.F.M. Torres, The contingent epiderivative and the calculus of variations on time scales, Optimization (in press). DOI: 10.1080/02331934.2010.506615.

27. M. Gürses, G.Sh. Guseinov, B. Silindir, Integrable equations on time scales, J. Math. Phys. 46(11), 113510, $22 \mathrm{pp}$, (2005).

28. S. Hilger, Analysis on measure chains-a unified approach to continuous and discrete calculus, Results Math. 18(1-2), 18-56, (1990).

29. V. Kac, P. Cheung, Quantum calculus, Springer, New York, (2002).

30. W.G. Kelley, A.C. Peterson, Difference equations. An introduction with applications Academic Press, Inc., Boston, MA, (1991).

31. V. Lakshmikantham, S. Sivasundaram, B. Kaymakcalan, Dynamic systems on measure chains, Mathematics and its Applications, 370 , Kluwer Acad. Publ., Dordrecht, (1996). 
32. A.B. Malinowska, N. Martins, D.F.M. Torres, Transversality conditions for infinite horizon variational problems on time scales, Optim. Lett. 5(1), 41-53, (2011).

33. A.B. Malinowska, D.F.M. Torres, Necessary and sufficient conditions for local Pareto optimality on time scales, J. Math. Sci. (N. Y.) 161(6), 803-810, (2009).

34. A.B. Malinowska, D.F.M. Torres, Strong minimizers of the calculus of variations on time scales and the Weierstrass condition, Proc. Est. Acad. Sci. 58(4), 205-212, (2009).

35. A.B. Malinowska, D.F.M. Torres, Natural boundary conditions in the calculus of variations, Math. Methods Appl. Sci. 33(14), 1712-1722, (2010).

36. A.B. Malinowska, D.F.M. Torres, Leitmann's direct method of optimization for absolute extrema of certain problems of the calculus of variations on time scales, Appl. Math. Comput. 217(3), 1158-1162, (2010).

37. A.B. Malinowska, D.F.M. Torres, The delta-nabla calculus of variations, Fasc. Math. 44, 75-83, (2010).

38. A.B. Malinowska, D.F.M. Torres, Delta-nabla isoperimetric problems, Int. J. Open Probl. Comput. Sci. Math. 3(4), 124-137, (2010).

39. A.B. Malinowska, D.F.M. Torres, The Hahn quantum variational calculus, J. Optim. Theory Appl. 147(3), 419-442, (2010).
40. A.B. Malinowska, D.F.M. Torres, Euler-Lagrange equations for composition functionals in calculus of variations on time scales, Discrete Contin. Dyn. Syst. 29(2), 577-593, (2011).

41. A.B. Malinowska, D.F.M. Torres, Backward variational approach on time scales with an action depending on the free endpoints, Z. Naturforsch. A (in press).

42. A.B. Malinowska, D.F.M. Torres, A general backwards calculus of variations via duality, Optim. Lett. (in press). DOI: 10.1007/s11590-010-0222-x.

43. N. Martins, D.F.M. Torres, Calculus of variations on time scales with nabla derivatives, Nonlinear Anal. 71(12), e763-e773, (2009).

44. N. Martins, D.F.M. Torres, Noether's symmetry theorem for nabla problems of the calculus of variations, Appl. Math. Lett. 23(12), 1432-1438, (2010).

45. E. Pawłuszewicz, D.F.M. Torres, Backward linear control systems on time scales, Internat. J. Control 83(8), 1573-1580, (2010).

46. J. Seiffertt, S. Sanyal, D.C. Wunsch, HamiltonJacobi-Bellmam equations and approximate $d y$ namic programming on time scales, IEEE Trans. Syst. Man Cybern. B 38(4), 918-923, (2008).

47. B. van Brunt, The calculus of variations, Universitext, Springer-Verlag, New York, (2004). 